\title{
CONOCIMIENTO, CERTEZA, DUDAS Y VIAJES A LA LUNA. VARIACIONES SOBRE UN TEMA WITTGENSTEINIANO
}

\author{
Isabel G. Gamero \\ IdIHCS, Universidad Nacional de la Plata, Conicet, Argentina \\ igamero@conicet.gov.ar
}

\section{RESUMEN}

En este artículo trato de comprender el valor de verdad de una serie de proposiciones, relacionadas con la posibilidad de viajar a la Luna, que Wittgenstein y Moore entendieron como certezas, dando distintas comprensiones de este concepto. Este ejemplo es interesante porque cuando Neil Armstrong alunizó en 1969, cambió el valor de verdad de algunas de las proposiciones que Wittgenstein y Moore entendieron como certezas y porque en la actualidad sigue habiendo personas que se muestran escépticas con la realidad de este viaje espacial. A partir de estos hechos, preguntaré si las certezas son proposiciones empíricas que aún no han sido demostradas, así como cuáles serían las evidencias que se podrían dar para demostrar la verdad o falsedad de proposiciones como estas. Para responder a estas preguntas y aclarar el concepto de certeza, en la primera parte del artículo contrastaré las comprensiones de Wittgenstein y Moore, y en la segunda parte trasladaré la reflexión sobre las certezas a un contexto cotidiano y familiar, siguiendo un método wittgensteiniano.

Palabras clave: creencia, evidencia empírica, escepticismo, lenguaje cotidiano, prueba.

\section{KNOWLEDGE, CERTAINTY, DOUBTS AND TRAVELS TO THE MOON. VARIATIONS ON A WITTGENSTEINIAN THEME}

\section{Abstract}

In this article, it is going to be analysed the truth value of some propositions, referred to the possibility of travelling to the Moon, which Moore and Wittgenstein defined as certainties, although they understood differently this concept. The main interest of this kind of certainties is that their truth value changed when Neil Armstrong landed into the Moon in 1969. Furthermore, some people still maintains skeptical positions about this spacial travel. Therefore, here it is going to be asked whether the certainties are empiric propositions that have not been proved yet and which are the evidences that might be given to prove the truth or falsity of these propositions. In order to answer these questions and clarify what it is a certainty, in the first part of the article I will contrast Wittgenstein's and Moore's concepts; in the second one, I will move the argumentation about this kind of certainties to an everyday and familiar context, following a Wittgensteinian method.

KEYWORDs: belief, empirical evidence, skepticism, everyday language, proof.

DOI: http://doi.org/10.25145/j.laguna.2018.42.004

Revista Laguna, 42; julio 2018, pp. 59-77; ISSN: e-2530-8351 


\section{ABORDAJE TEÓRICO DEL PROBLEMA}

\subsection{Caracterización de las certezas en las obras de Moore y Wittgenstein}

En este artículo voy a aclarar el concepto de certeza que desarrollaron, con diferencias, Wittgenstein y Moore a mediados del siglo xx, como un medio para dirimir cuál de estas dos comprensiones resulta más adecuada para explicar el papel que juegan las certezas en los usos cotidianos del lenguaje. Para delimitar el tema sólo trataré un tipo específico de certezas: las referidas a la permanencia del ser humano sobre la superficie terrestre o la posibilidad de ir al espacio exterior, por ejemplo, con un viaje a la Luna. He elegido este tema por ser uno de los ejemplos privilegiados por Wittgenstein y Moore para explicar las certezas y porque presenta dos dificultades: ambos autores consideraron que expresiones como «el ser humano nunca se ha separado de la Tierra para ir a la Luna» eran certezas y, sin embargo, el valor de verdad de esta expresión (y otras similares) ha cambiado con los avances técnicos aeroespaciales que posibilitaron el viaje a la Luna de Armstrong en 1969. La segunda dificultad es que pese a este cambio, hoy en día sigue habiendo personas escépticas que no creen que el alunizaje fuera real y dudan del valor de verdad de estas expresiones. A través del desarrollo de estas dificultades, pretendo aclarar el concepto de certeza, en los dos autores mencionados, y definir mejor cuál es la relación entre certeza y lenguaje cotidiano; con esta expresión me refiero a un lenguaje no especializado ni científico, sino al que usamos en nuestro día a día.

Comenzaré destacando algo bien sabido: desde 1949 hasta pocos días antes de su fallecimiento, Ludwig Wittgenstein escribió una serie de apuntes que, posteriormente, fueron recopilados por Elizabeth Anscombe y George H. von Wright, en lo que se entiende como la última obra del autor, Sobre la certeza. Pese a la multitud de temas y líneas de pensamiento que se pueden encontrar en este libro, existe una constante o hilo conductor, basado en la crítica que Wittgenstein dirigió al concepto de certeza que Moore propuso en 1925, en el ensayo Defensa del sentido común; como alternativa a este concepto, Wittgenstein propuso una nueva comprensión de las certezas. A continuación, desarrollaré estas dos comprensiones y las constrastaré. Para Moore, las certezas son las bases o piezas elementales del conocimiento humano que resultan verdaderas e indudables, en todos los casos y para todas las personas. Los ejemplos que da de certezas son expresiones como "esta es mi mano", "todos los seres humanos tenemos cuerpo» 0 «nunca me he separado de la superficie terrestre» ${ }^{1}$. Considera que las certezas son "verdades palmarias" ${ }^{2}$, incuestionables e indudables y desarrolla una prueba para mostrar que es imposible dudar de ellas y refutar así cualquier planteamiento escéptico que pretenda cuestionarlas. El peso de esta prueba radica en una acción, acompañada de una expresión: Moore levanta su mano y

\footnotetext{
1 Moore, George H.: «Defensa del sentido común», en Defensa del sentido común y otros ensayos, Taurus, Madrid, 1972, p. 50.

2 Ibidem. p. 52.
} 
dice "esta es mi mano $»^{3}$ y considera, así, que ha mostrado una certeza innegable e imposible de dudar o refutar. En un segundo momento, extiende esta constatación a todos los cuerpos humanos, considerando que si es verdadera para él, lo será para cualquiera que levante su mano y afirme que se trata de su mano. Moore considera entonces que su prueba para mostrar la verdad de las certezas es irrefutable, para toda la humanidad, ya que si alguien, por ejemplo un autor escéptico, tratara de dudar de esta prueba acabaría contradiciéndose, ya que o bien la persona que duda no tiene, en realidad, cuerpo, manos o no está sobre la superficie terrestre, por lo que no podría mantener esta opinión, o bien sí tiene cuerpo, manos y está sobre la superficie terrestre, por lo que su duda sobre tener cuerpo o estar sobre la superficie terrestre carece de sentido o se trata de algo incomprensible, de una incoherencia o de un error ${ }^{4}$.

Es resumen, Moore mantiene una concepción incuestionable, invariable, universal de las certezas, que cree demostrables gracias a la prueba explicada, y las entiende como contenidos más ciertos y seguros que las verdades empíricas, que son contingentes y pueden variar. En estas consideraciones de indudabilidad y demostrabilidad radican las diferencias entre la comprensión de Moore y la de Wittgenstein. Este segundo autor, por el contrario, no entiende que las certezas sean verdaderas, incuestionables ni demostrables, sino que las define como un conjunto de proposiciones que parecen ser conocidas por todos, sin que podamos explicar cómo las sabemos ( $\mathrm{SC} \$ 84)^{5}$ y que, aunque no sea posible probarlas ni refutarlas, si no se cuestionan, posibilitan el conocimiento humano. Es decir, para Wittgenstein las certezas no son incuestionables sino que no están cuestionadas, porque no tenemos motivos para dudar de ellas (SC $\$ 4, \$ 92$ ) y no entenderíamos muy bien a quien, dentro de nuestro juego de lenguaje, pretendiera ponerlas en duda (SC \$32, $\$ 108)$. Aunque estas proposiciones sean bastante diferentes entre $s^{6}{ }^{6}$, según Wittgenstein, lo que tienen en común es que juegan un papel «muy interesante» en el sistema de nuestros juicios empíricos: los limitan y, al mismo tiempo, los posibilitan (SC \$138). Es decir, partiendo de la imposibilidad de fundar o demostrar de modo

${ }^{3}$ Moore, George H.: «Prueba del mundo externo», en Defensa del sentido común y otros ensayos, op. cit, p. 178.

${ }_{4}$ Moore, George H.: «Defensa del sentido común», Defensa del sentido común y otros ensayos, op. cit, pp. 67-69.

5 Ludwig Wittgenstein, Sobre la certeza, Gedisa, Barcelona, 1998, \$84. De aquí en adelante, citaré del modo canónico Sobre la certeza de Wittgenstein, esto es, en el cuerpo del texto, entre paréntesis, como «SC» seguido del aforismo correspondiente.

${ }^{6}$ No existe una catalogación completa y exhaustiva de las certezas y sus tipos, aunque Danièle Moyal-Sharrock (Understanding Wittgenstein's 'On Certainty', Palgrave Macmillan, London, 2007, pp. 102-103) ha intentado sistematizarlas y las ha dividido en cuatro categorías (lingüísticas, personales, locales y universales). Sin embargo, estas categorías se intersecan. Por ejemplo, las certezas analizadas en este artículo pueden ser, según el caso, el contexto o la forma de enunciación, certezas personales («sé con seguridad que nunca he estado en la Luna»), locales («estoy sobre la superficie terrestre») o incluso universales (la generalización de las dos anteriores y su adscripción a toda la humanidad). 
definitivo el conocimiento humano 7 , Wittgenstein adujo que ciertas proposiciones, las certezas, funcionan como el trasfondo que viene dado a los miembros de un juego de lenguaje que les permite distinguir lo verdadero de lo falso, sin que se pueda probar su verdad o falsedad (SC \$94). Esto se debe a que si lo verdadero es lo que tiene fundamentos, lo que puede ser probado o demostrado con evidencias, ¿cómo se puede saber el valor de verdad de los fundamentos, de lo que nos permite probar o demostrar lo que conocemos? Para este autor, el fundamento, en sí, no es ni verdadero ni falso (SC \$205), sino que está formado por certezas que, sin ser verdaderas o falsas, demostrables o indemostrables, no se han de cuestionar para que se dé conocimiento empírico y para que podamos formular con claridad nuestras preguntas y nuestras dudas.

Además, las certezas no son un saber implícito que pueda ser explicitado, razonado o probado, sino una actitud (SC \$404) o práctica compartida por distintos hablantes, miembros de un mismo juego de lenguaje. En la base del conocimiento no se dan verdades ni falsedades sino un «modo de actuar sin fundamentos» (SC \$110). Las certezas son, entonces, prácticas o actitudes que se originan en la acción humana; en palabras de Wittgenstein: «Es nuestra actuación la que yace en el fondo del lenguaje» (SC \$204). Cabe reformular esta idea manteniendo que para que haya acción y comunicación entre los seres humanos es imprescindible que ciertas dudas no se planteen. Por ejemplo, si alguien está usando una mesa, es difícil que dude de la existencia de ese mueble (SC \$75), confirmándose así la certeza de que algunos objetos existen. Por otro lado, enunciar una certeza como: «sé que los objetos existen» resultaría extraño, difícil de comprender, no sería un uso cotidiano del lenguaje.

Por último, aunque una certeza pueda ser formulada, aislada y analizada por separado, por ejemplo para fines clarificatorios (esta sería una de las tareas de la filosofía, según Wittgenstein, IF\$ 125), en general las certezas no se presentan como axiomas aislados, ni resultan evidentes cuando se dan por separado, sino que constituyen un sistema de proposiciones o totalidad de juicios (SC $\$ 140$ ) que aceptamos porque sus «consecuencias y premisas se sostienen recíprocamente» (SC \$141-142) y que se dan interrelacionadas, formando una estructura convincente, que Wittgenstein denomina $«$ nido ${ }^{8}(\mathrm{SC} \$ 225)$.

\footnotetext{
${ }^{7}$ Esta imposibilidad es un clásico de la epistemología, que toma la forma de un trilema, conocido como «trilema de Münchhausen» y teorizado por Hans Albert en Treatise on Critical Reason (New Jersey, Princeton UP, 1985), según el cual la fundamentación del conocimiento es imposible porque se da o una regresión infinita o un círculo lógico o un corte arbitrario y dogmático en el razonamiento. Esta dificultad ya fue destacada por los escépticos griegos, como Diógenes Laercio. Las soluciones que se han intentado dar en la historia de la filosofía a este dilema oscilan, grosso modo, entre la aceptación de que el conocimiento humano no se puede fundamentar, por lo que es falible y poco seguro (en la corriente escéptica) y la búsqueda de una base incuestionable e innegable del conocimiento (en las corrientes empirista y racionalista). La certeza según Moore sería una base innegable del conocimiento; mientras que la de Wittgenstein supone una comprensión alternativa, ni escéptica ni empirista.

${ }^{8}$ Cabe destacar que el término usado por Wittgenstein en el original para explicar a las certezas es Nest (nido), no Net (red), habiendo un error en la traducción.
} 


\subsection{Preguntas de esta investigación}

Como ya se ha explicado, uno de los ejemplos de certeza que dieron Moore y Wittgenstein es el hecho de que ningún ser humano se haya separado de la Tierra para ir a la Luna. Para el primer autor, esto era incuestionable y demostrable'; para Wittgenstein, si en nuestro juego de lenguaje alguien mantuviera que había viajado a la Luna, se alteraría el funcionamiento habitual de tal juego de lenguaje y se abrirían demasiadas preguntas sin respuesta, ya que ¿cómo habría logrado esta persona sustraerse a la fuerza de la gravedad, sobrevivir sin oxígeno fuera de la atmósfera y viajar hasta el satélite sin perecer durante el trayecto? Y es más, si alguien asegurara haber logrado esto y saberlo, pero no pudiera responder a las preguntas planteadas, esta persona se encontraría, según Wittgenstein, intelectualmente muy alejada de nosotros, nos costaría comprenderla ${ }^{10}$.

Sin embargo, la técnica aeroespacial ha avanzado y, como consecuencia, el estatus de este tipo de certezas ha variado. Es decir, cuando Armstrong alunizó y dejó la primera huella humana sobre este satélite en 1969, la certeza (posiblemente universal) que aducía que ningún ser humano había ido a la Luna se convirtió en una proposición falsa y la certeza personal o subjetiva que lleva a alguien a decir «sé que nunca he estado en la Luna» pasó a ser una proposición verificable, cuyo valor de verdad depende del sujeto enunciador. Por lo tanto, es posible mantener que las proposiciones relacionadas con los viajes a la Luna han dejado de ser certezas, para pasar a formar parte del cuerpo de conocimiento empírico, formulable en proposiciones que pueden ser probadas o demostradas con evidencias. Visto este cambio del rol de ciertas proposiciones que Wittgenstein y Moore entendieron, con sus diferencias, como certezas pero que, con el avance de la técnica y del conocimiento científico, parecen haberse convertido en proposiciones empíricas, ¿cabe entonces sostener que las certezas referidas a los límites humanos en relación con el espacio exterior (como «ningún ser humano ha vivido en la Luna» o "nadie ha estado en Júpiter») se van a integrar progresivamente en el cuerpo de proposiciones empíricas y verificables? Es decir, ¿es posible que esta serie de certezas sean, en realidad, proposiciones empíricas que aún no han sido probadas o refutadas, aunque lo serán en el futuro? ¿Podríamos entonces considerar que Wittgenstein y Moore se equivocaron al entender que proposiciones como «sé que no he estado en la Luna» y «ningún ser humano ha estado nunca en la Luna» eran certezas? En ese caso, ¿resulta más adecuada la comprensión de Moore ya que, aunque sus ejemplos de certezas sobre el espacio exterior estuvieran errados, acertó al proponer la posibilidad de probar y demostrar las certezas? Pero si aceptamos la prueba de Moore, ¿qué diferenciaría entonces una certeza de una proposición empírica? En definitiva, ¿es posible que con el avance de la técnica y del conocimiento científico las certezas desaparezcan?

9 Cf. supra o Moore, George H.: «Defensa del sentido común», Defensa del sentido común y otros ensayos, op. cit., p. 67 y ss.

${ }_{10}$ Estas preguntas y consideraciones son variaciones de Wittgenstein, Sobre la certeza $\$ 108$. 


\subsection{Contrastes entre las comprensiones de Wittgenstein y Moore}

Como primera anticipación de respuesta a estas preguntas, y como un modo de seguir aclarando el concepto de certeza de estos autores, cabe destacar que, tanto en las Investigaciones como en Sobre la certeza, Wittgenstein adujo que Moore se equivocaba cuando intentaba dar un listado exhaustivo de todas las certezas demostrables y decir que las sabía y las podía demostrar ${ }^{11}$ (SC \$6), ya que no existe una distinción precisa entre certezas y proposiciones empíricas y es necesario acudir al contexto para saber cuándo una proposición se da controlada por la experiencia (esto es, es una proposición empírica) o como regla de control (esto es, una certeza) (SC $\$ 97,98)$. Moore se equivocó al no tener en cuenta, continúa Wittgenstein, que las certezas pueden cambiar, ya sea por el paso del tiempo (SC \$96, \$236), por los distintos usos que se les dé (IF $\$ 23$ ), por el juego de lenguaje donde se encuentren o la finalidad con la que se hayan enunciado (IF $\$ 410-411$ ). Es decir, dependiendo del caso, del uso o del contexto (y de otras muchas variantes...) una misma proposición puede funcionar como empírica o como certeza. Por ejemplo, la proposición «sé que esto es un árbol» (otro de los ejemplos de certeza de Wittgenstein, SC \$349-350) puede ser una aclaración en una discusión sobre botánica o una orientación en medio de un banco de niebla, y entonces funcionaría como una proposición empírica y corroborable; pero también puede ser la explicación que se le da a alguien que no habla correctamente castellano o lo que diría un niño que acaba de aprender el uso de ese término, y en esos casos funcionaría como regla de control o certeza. A través de estos ejemplos, vemos cómo resulta necesario conocer el contexto y la finalidad de una frase para entenderla plenamente y saber si se trata o no de una certeza, porque podría suceder, como narra Wittgenstein, que un hombre estuviera en un jardín y no dejara de repetir «sé que esto es un árbol», mientras alguien que lo observa desde fuera creyera que este hombre estaba loco, cuando en realidad se trataba un filósofo reflexionando sobre el uso del verbo «saber» (SC \$467). Con esta explicación pluralista, contextual y cambiante de las certezas, Wittgenstein problematiza y critica la comprensión universal, absoluta y sin excepciones que propuso Moore, ya que incluso se pueden plantear casos donde las expresiones de la prueba de Moore, como «sé que tengo manos» o "sé que esta es mi mano", no resulten tan seguras. Por ejemplo: un hombre despierta en la cama de un hospital con los brazos completamente vendados, no sabe qué le ha sucedido, no recuerda nada. ¿Y si le han operado y le han amputado una mano, como le sucedió al hermano de Wittgenstein, Paul?, ¿y si le han hecho un trasplante y ahora tiene las manos de otra persona o una mano ortopédica? ${ }^{12}$. Escenarios como estos, quizás extremos pero posibles, muestran que la seguridad completa y para todos los casos que Moore daba a las certezas no se sostiene.

11 Énfasis de Wittgenstein.

12 Variaciones de Sobre la certeza $\$ 10$ y $\$ 23$. 
Volviendo a nuestro ejemplo, cabe pensar las diferentes funciones que puede cumplir la proposición "sé que he estado en la Luna» en distintos contextos; por ejemplo, en una evaluación psicológica, en las declaraciones a prensa de un astronauta, al despertar de un sueño, en una clase de filosofía, o en lo que nos dice alguien que estaba muy despistado y no nos prestaba atención. Se pueden diferenciar entonces los usos de esa proposición que son corroborables empíricamente (el caso del astronauta); los usos cotidianos donde, a pesar de existir numerosas evidencias en su contra y con independencia de la verdad de la aserción, la entendemos, ya que no es una proposición empírica verificable sino una metáfora (los sueños y los despistes); los usos donde funciona como certeza (la clase de filosofía); y los usos que aún resultarían anómalos o extraños, cercanos a la locura, a no ser que se tratara de un uso poco común con una finalidad específica y explicable (por ejemplo, un nuevo tratamiento psicológico donde se deja al paciente dar rienda suelta a sus ilusiones).

Esta explicación plural, contextual y cambiante de las certezas de Wittgenstein resulta consecuente, además, con la segunda etapa del pensamiento de este autor en la que rechazó dar una explicación totalitaria y exclusiva del funcionamiento del lenguaje y el conocimiento humano, abriendo espacio para un pluralismo de explicaciones y comprensiones. Como es bien sabido, Wittgenstein criticó la concepción unitaria del conocimiento y el lenguaje que él mismo había propuesto en el Tractatus, según la cual la totalidad de las proposiciones verdaderas era la ciencia natural en su conjunto ( $\mathrm{T} \$ 4.11)^{13}$; mientras que las restantes disciplinas o cualquier aserción no verificable empíricamente debían ser descartadas por suponer un sinsentido ( $c f$. $\mathrm{T} \$ 4.461 s s$. y $\$ 6.4 s s$.$) . A diferencia de esta comprensión, a partir de los apuntes$ que componen el Cuaderno azul, pero sobre todo en las Investigaciones filosóficas y en Sobre la certeza, Wittgenstein criticó los intentos de dar una única concepción del conocimiento y del lenguaje, según la cual existe una única forma general de la proposición que se corresponde con la realidad (IF $\$ 111-114)^{14}$, y recuerda, por el contrario, que existen «innumerables géneros diferentes de empleo de todo lo que llamamos 'signos', 'palabras', 'oraciones'” (IF \$23).

Siguiendo con el pluralismo y la imposibilidad de dar una explicación general y unitaria del funcionamiento del lenguaje, para Wittgenstein estos «innumerables géneros diferentes» no vienen dados de antemano ni se mantienen siempre igual, sino que se originan en los distintos usos que se hacen del lenguaje y en los distintos contextos donde se usan las oraciones (IF $\$ 43$ ). Además, también insistió en que los distintos usos del lenguaje no poseen nada en común que la filosofía pueda abstraer para dar una explicación general, sino que estos usos se dan de formas distintas, y cambiantes, en ocasiones parecidas entre sí, como los parecidos familiares o la superposición de hilos que componen una madeja (IF \$65-67). Y también mantuvo

13 Wittgenstein, Ludwig: Tractatus Logico Philosophicus, Alianza, Madrid, 1999, \$4.11. Citaré el Tractatus en el cuerpo del texto, entre paréntesis, como «T» seguido del aforismo correspondiente.

${ }_{14}$ Wittgenstein, Ludwig: Investigaciones filosóficas, Crítica, Barcelona, 2008, \$111-114. Citaré esta obra en el cuerpo del texto, entre paréntesis, como «IF» seguido del aforismo correspondiente. 
que las certezas cambian con el tiempo (SC \$96, \$236), por lo que sería admisible que una certeza como "ningún ser humano ha ido nunca a la Luna» dejara de serlo y se convirtiera en falsa como, de hecho, ha sucedido.

\subsection{NueVAS PREgUnTAS Y EXPLICACiÓN DE UN MÉTODO WITTGENSTEINIANO}

Tras esta comparativa entre las obras de Moore y Wittgenstein, se puede comenzar a anticipar que la comprensión plural y cambiante de las certezas que da Wittgenstein resulta más adecuada que la absoluta e invariable de Moore, en tanto se asemeja más a cómo usamos los seres humanos el lenguaje en nuestra vida cotidiana. Piénsese, por ejemplo, en lo extraño que resultaría que una persona fuera enumerando todo el rato las cosas que sabe, siguiendo la prueba de Moore. Sin embargo, aún no se ha llegado a responder del todo a la pregunta formulada anteriormente que plantea que las certezas relacionadas con la presencia del ser humano en el espacio exterior son proposiciones empíricas que van a ser probadas y demostradas progresivamente, conforme la ciencia aeroespacial avance, lo que podría llegar a causar la desaparición de este tipo de certezas, al integrarse en el cuerpo de conocimiento empírico demostrable. Esto es, por mucho que quepa pensar en distintos usos de las certezas, en distintos contextos y con distintas finalidades, parece quedar claro que, a partir del alunizaje de Armstrong, la proposición «nadie ha estado en la Luna» es falsa y la frase "sé que he estado en la Luna» no es una certeza sino una proposición empíricamente verificable que será verdadera o falsa dependiendo de quién la enuncie. Y aunque quepa pensar y entender usos metafóricos, psicológicos, filosóficos o poéticos de proposiciones como «no sé si he estado en la Luna» o "creo que anoche volé a la Luna», esta posibilidad no invalidaría el hecho de que algunos hombres ${ }^{15}$ hayan ido a la Luna y otros no. Esto es, en la actualidad se puede comprobar la verdad empírica de las proposiciones relacionadas con el espacio exterior que Wittgenstein y Moore entendieron como certezas y que ahora parecen haberse convertido en proposiciones empíricas verificables.

Ahora bien, esta posible conversión de las certezas en proposiciones empíricas corroborables resulta semejante a la comprensión positivista del conocimiento que Wittgenstein mantuvo en el Tractatus y se distancia, con creces, del concepto de certeza que el filósofo vienés definió en su segunda etapa y que se acaba de explicar. ¿Por qué se tomó entonces Wittgenstein tantas molestias para criticar su primera obra, así como la explicación de Moore? Para responder a estas preguntas y aclarar el concepto de certeza wittgensteiniano, a diferencia de lo que él mismo mantuvo en el Tractatus y de la comprensión absoluta de las certezas de Moore, recurriré a lo que

15 Aquí pongo «hombre» a propósito ya que, hasta el momento, las únicas doce personas que han pisado la Luna son de género masculino. 
el segundo Wittgenstein entiende como uno de los métodos de la filosofía ${ }^{16}$, dirigido a reconducir las palabras de su uso metafísico a su empleo cotidiano (IF \$116) y a realizar exámenes gramaticales para eliminar malentendidos sobre el uso de las palabras (IF \$90). Según Wittgenstein, estos malentendidos se pueden producir (entre otras posibilidades) cuando se intenta dar una forma general de la proposición, que pretenda ser más exacta que las demás o válida en todos los casos. Para evitar esta tendencia (que aparecía tanto en su Tractatus como en la comprensión absoluta de las certezas de Moore), Wittgenstein recomendaba ir a los usos cotidianos de las palabras y buscar o inventar casos intermedios (IF \$122), para mostrar las confusiones que se dan en las comprensiones absolutas y generales, tan propias de la filosofía, o cuando el lenguaje funciona "en el vacío" y no tiene en cuenta las distintas finalidades y distinciones de los usos del lenguaje (IF \$132). De este modo, si nos encontramos ante una expresión que indica que las cosas siempre son de un modo determinado («las cosas son así y asá», en palabras de Wittgenstein), cabe preguntar cómo se usa esa expresión en el lenguaje cotidiano, en qué contexto se puede aplicar, para poder comprenderla, contextualizarla y aclararla (IF \$134).

Siguiendo este método, en la segunda parte de este artículo trasladaré las proposiciones relacionadas con los viajes a la Luna a un contexto cotidiano, para ver qué evidencias se admiten para comprobar esas proposiciones y si sigue habiendo certezas en esos casos o si, por el contrario, las proposiciones que parecían ser certezas se han integrado en el cuerpo del conocimiento empírico.

\section{ANÁLISIS DE UN CASO COTIDIANO: UN ALMUERZO EN CASA DE LA ABUELA}

\subsection{Presentación}

Imaginemos una escena cotidiana; por ejemplo, un almuerzo familiar en la casa de la abuela, cualquier día menos el 28 de diciembre. De repente, uno de nuestros primos se levanta y nos dice: «Sé que he estado en la Luna». Su expresión nos causaría extrañeza, parecería fuera de contexto y rayana en la locura, nos costaría creerle; sin embargo, estamos en el siglo XxI, los viajes espaciales parecen ser una realidad, algunos astronautas dicen que han ido al espacio y hay personas millona-

16 Este no es el único método o la única tarea de la filosofía, sino que vuelve a darse una pluralidad de métodos, dependiendo del caso y de las necesidades. Según Wittgenstein la filosofía ha de desentrañar malentendidos lingüísticos y conceptuales, pero estos pueden ser muy distintos (por exceso de abstracción, por usar símiles no válidos, por tratar de explicar diferentes fenómenos con una misma teoría o disciplina...) y los métodos o terapias que se pueden aplicar para resolver tales malentendidos también varían. Véase el listado de distintos problemas y distintos métodos que Oskari Kuusela encuentra en la obra del segundo Wittgenstein (The Struggle Against Dogmatism. Massachusetts, Harvard University Press, 2008, p. 270). 
rias que pagan para hacer viajes espaciales ${ }^{17}$. Por estos motivos, podríamos creer a nuestro primo aunque quizás le pediríamos evidencias, pruebas, que nos explicara cómo lo hizo y por qué no nos lo había contado antes. Es decir, si nos tomásemos en serio su aserción (y no pensáramos, por ejemplo, que se trataba de una broma, de un exceso de alcohol o de un momento de locura), le preguntaríamos cuándo fue, cómo llegó allí, cómo lo pagó, por qué no nos lo había contado antes y por qué no hizo alguna foto y la publicó en Facebook o Instagram ${ }^{18}$.

Aunque nuestro primo pudiera responder a todas estas preguntas con explicaciones coherentes (no lo dijo antes porque pensaba que no lo íbamos a creer, ganó el viaje en un concurso de ciencia, era un estudio científico ultrasecreto o un proyecto piloto para investigar cómo responden las personas no preparadas en un viaje espacial, estaba prohibido tomar fotos...) o incluso si nos diera evidencias (el contrato que firmó con la NASA, su identificación para entrar a la nave, su traje de astronauta, algunas fotos, porque aunque estaba prohibido logró hacer un par a escondidas...), posiblemente seguiríamos sin creerlo del todo, desconfiaríamos de las evidencias y lo miraríamos con extrañeza. Pero si persistiera en sus explicaciones, no hablara de otra cosa y trajera a sus amigos astronautas a la siguiente comida familiar y nos enseñaran sus fotos de grupo en la nave espacial, algunos comenzarían a creerlo, mientras que otros seguirían pensando que era todo un montaje, no se fiarían de las evidencias y seguirían pensando que se trataba de una mentira, un caso de locura colectiva o que nos quería engañar por algún motivo concreto. Entonces, ante la declaración "sé que he estado en la Luna», en un contexto cotidiano y familiar, parecen darse las dos siguientes actitudes: por un lado, se puede entender la declaración de nuestro primo como una proposición verificable y pedir evidencias para probarla o desmentirla, es decir, mantener el modo habitual de corroboración del conocimiento empírico. Por el otro lado, está la actitud escéptica de quienes, pese a todas las evidencias empíricas que se puedan dar, siguen desconfiando (o directamente no creyendo) a quien mantenga esta aserción. Aquí también pueden aparecer dudas escépticas más radicales, como la de la abuela ${ }^{19}$, que nunca creyó el alunizaje y sostiene que la proposición «el hombre ha llegado a la Luna» es falsa.

En el siguiente apartado, analizaré estas dos posibles actitudes en relación con la comprensión de las certezas del segundo Wittgenstein; pero antes, contrastaré este ejemplo de la comida familiar y el primo astronauta con la comprensión absoluta e invariable de las certezas que da Moore para seguir mostrando (como

17 Por ejemplo, la compañía estadounidense Space X anunció en 2017 que dos personas, que preferían guardar su anonimato, ya se estaban preparando y entrenando para ser los primeros astronautas no profesionales que viajarían a la Luna en 2018, previo pago de una "cantidad significativa» de dólares, que tampoco se especificaba. Véase la noticia completa en este vínculo: http:// www.spacex.com/news/2017/02/27/spacex-send-privately-crewed-dragon-spacecraft-beyond-moonnext-year [última consulta: 29 de enero de 2018].

${ }_{18}$ Variaciones de SC $\$ 108$, adaptadas al presente y al ejemplo que estamos desarrollando.

19 Este ejemplo es real e inspirado en mi propia familia: una tía abuela sigue sin creer que el hombre haya ido a la Luna y desconfía también de la existencia de los dinosaurios. 
ya se aclaró en la primera parte de este artículo) que esta comprensión no resulta satisfactoria cuando se traslada a una situación cotidiana, donde el hecho de que algo sea o no una certeza puede cambiar, dependiendo del momento, del contexto y de la actitud de los miembros de ese juego de lenguaje. Es decir, si nuestro primo propusiera una prueba, en apariencia irrefutable, como la que propuso Moore para mostrar la existencia de su cuerpo, su aserción seguiría siendo susceptible de dudas, desconfianzas y cuestionamientos. Es decir, ¿cómo o cuál podría ser esta prueba irrefutable que mostraría, sin lugar a dudas, que realmente fue a la Luna? Las fotos pueden ser un montaje, las piedras, en apariencia lunares, pueden ser falsas o meteoritos hallados en la Tierra... Cualquier argumento o prueba que nos diera nuestro primo puede ser dudado o cuestionado y las dos posibilidades mencionadas se pueden seguir dando: por un lado, se le pueden pedir a nuestro primo pruebas empíricas y contrastables para corroborar la verdad de su afirmación; y la prueba absoluta de Moore no valdría en este caso, ya que al entender la certeza como una proposición empírica, su verdad será contingente, esto es, dependerá del caso, del contexto o del sujeto que la enuncie. La afirmación de nuestro primo no sería entonces una certeza, en el sentido de Moore, sino una verdad empírica contrastable, verificable o falsable. Por otro lado, y pese a todas las pruebas que nos diera, podríamos seguir dudando de la afirmación de nuestro primo, manteniendo posturas escépticas $y$, de nuevo, la prueba de Moore no sería suficiente ni significativa para convencer a nuestra abuela escéptica.

En conclusión, la prueba para demostrar las certezas que propuso Moore no funciona cuando se traslada a un contexto cotidiano como el del ejemplo elegido. Si se entienden las certezas en el sentido del segundo Wittgenstein, se puede analizar este ejemplo en los dos escenarios posibles (verificación empírica o escepticismo), para de este modo tratar de responder a la pregunta planteada sobre si las certezas sobre el espacio exterior van a desaparecer al convertirse en proposiciones empíricas contrastables.

\subsection{Dos Posibles ACtitudes}

\subsubsection{La certeza entendida como objeto de corroboración empírica}

Si se entiende la proposición «sé que he estado en la Luna» como empírica y verificable, entonces se pueden pedir evidencias para demostrarla y su comprobación dependerá de la confianza que tengamos en nuestro primo y también en la ciencia y la técnica aeroespacial. Es decir, si creemos que en otros casos se han dado viajes a la Luna será más probable que creamos a nuestro primo; pero incluso en ese caso, le pediríamos pruebas, evidencias y explicaciones para comprobarlo. En este caso, la proposición «sé que he estado en la Luna» funciona como una proposición empírica verificable. ¿Nos encontraríamos entonces ante la posibilidad, sugerida anteriormente, de que las certezas van a ser progresivamente eliminadas y sustituidas por saber demostrable con evidencias empíricas, propio de la ciencia?

No necesariamente, ya que según la explicación de las certezas que dio el segundo Wittgenstein, no se puede comprobar todo: cuando comprobamos algo, 
pedimos o damos evidencias, ya lo hacemos confiando en algo más, presuponiendo algo que no se comprueba. Es decir, cuando alguien hace un experimento en un microscopio, ¿comprueba también que ese microscopio es el mismo que usó el día de antes y que no desaparece cuando no lo usa? (SC \$163). Esta comprobación no parece resultar necesaria y, sin embargo, no parece ser un conocimiento empírico, sino una muestra de confianza o de lo que Wittgenstein llama certeza. El autor explicó este hecho con el ejemplo de Lavoisier (SC \$167), quien realizaba experimentos en su laboratorio y obtenía conclusiones, pero no se cuestionaba la existencia de los aparatos y las sustancias que estaba utilizando. ¿Eso quiere decir que este químico sabía esto del mismo modo que sabía que si mezclaba cualquier sustancia orgánica con permanganato se iba a producir una explosión? No, se trata de distintas formas de saber que, según Wittgenstein y como ya se explicó en la primera sección, no se pueden delimitar claramente, de antemano y para siempre, sino que dependen del caso y del uso o la finalidad de estas proposiciones. En el ejemplo de la química, los riesgos de mezclar permanganato con sustancias orgánicas son parte de un saber empírico demostrable, mientras que la existencia de esta sustancia o la constatación de que no va a desaparecer cuando no se está usando son certezas de este juego de lenguaje. Esto es, Lavoisier (y cualquiera de nosotros) ya tiene su propia imagen del mundo, adquirida desde la infancia, que es el fundamento evidente de su investigación y que, por ello mismo, ni siquiera se menciona (SC \$167). Una de las certezas de este juego de lenguaje de la ciencia es que siempre que se mantengan las mismas condiciones en un laboratorio, los resultados de un experimento serán iguales; se confía así en la ley de la inducción ${ }^{20}$, pero de ahí no cabe decir «sé que la ley de la inducción es verdadera» (SC \$500).

Es decir, en la base de cada comprobación empírica hay un límite que se debe a la imagen del mundo de cada uno, que subyace de modo implícito e incuestionado, y ha sido adquirida desde la infancia (o desde la adquisición de un nuevo juego de lenguaje). Desconocemos si la imagen del mundo es verdadera, no nos hemos convencido en ningún momento de que sea la correcta porque la tenemos con anterioridad a saber qué significa "corrección", se trata del trasfondo que nos viene dado y sobre el que distinguimos lo verdadero y lo falso (SC \$94). Esta imagen nos orienta sobre qué fuentes son más fiables (SC \$143), cuándo se pueden pedir evidencias de un conocimiento y cuándo no, dónde está el límite de cada comprobación, así como qué es preciso investigar y qué no, qué se puede dudar y qué no (SC \$472). Y esta estructura de contenidos asumidos y no cuestionados serían las certezas. Por ejemplo, si estudiamos el proceso de formación de las montańas antiguas, no nos planteamos si la Tierra comenzó a existir al mismo tiempo que nosotros sino que asimilamos que tiene, al menos, tantos ańos de antigüedad

${ }^{20}$ Este argumento de Wittgenstein es similar al ejemplo clásico del escepticismo humeano según el cual no podemos decir «sé que el Sol saldrá mañana»; sin embargo, tampoco podemos dudar de ello. Véase la Investigación sobre el entendimiento humano (Madrid, Istmo, 2004). Sobre la cercanía del pensamiento humeano y wittgensteiniano en torno a este concepto de certeza, puede leerse el primer capítulo de Escepticismo y naturalismo, de Peter Strawson (Madrid, Antonio Machado Libros, 2003). 
como las primeras formaciones montańosas. Creemos en eso (Wittgenstein llega a decir «nos lo tragamos», SC $\$ 143$ ) por la confianza que tenemos en los maestros y en los libros de texto (SC \$263). Es decir, en la base del conocimiento (inclusive el científico) se dan una serie creencias, las certezas, que forman el sustrato de nuestras comprobaciones y afirmaciones (SC \$162), y están compartidas por los miembros de una misma comunidad (SC \$288). Si alguien no creyera en este sustrato o planteara dudas y preguntas que lo pusieran en cuestión; por ejemplo, si, estudiando historia, alguien dudara si la Tierra existía en la época de Napoleón, nos costaría entenderlo, no sabríamos qué cuenta como evidencia para él (SC \$185).

Las certezas no son sólo proposiciones o creencias no cuestionadas, sino que también constituyen un modo de actuar común; por ejemplo, y como ya se ha mantenido, al aprender a realizar procedimientos científicos, también se aprende, de modo implícito, que no resulta necesario comprobar constantemente el funcionamiento o la calibración de los aparatos utilizados y que no desaparecen cuando no se usan. De nuevo, este tipo de saber no se debe a ninguna evidencia, sino a razones prácticas, es decir, cuestionarlo todo ralentizaría las investigaciones, impediría avanzar y no produciría conocimiento (SC \$33). Cabría imaginar, sin embargo, a un científico que comprobara incesantemente el funcionamiento de sus aparatos; en este caso, podríamos considerar que su conducta es ligeramente obsesiva y quizás criticarlo por ello. También podríamos preguntarnos si su seguridad sería mayor que la nuestra, por haber comprobado algo cien veces. Y Wittgenstein responde a esta pregunta que posiblemente no, el científico estaría tan seguro (o tan poco seguro) como cualquiera de nosotros (SC \$77). Es decir, toda comprobación empírica tiene un límite, aunque no podamos establecerlo con claridad porque, entre otros motivos, este límite no se debe a ningún argumento ni teoría, no es proposicional y descriptible, sino una práctica, aprendida en cada comunidad, un modo de hacer común o una forma de actuar sin fundamentos (SC \$110).

Si trasladamos estas consideraciones a nuestro ejemplo, cabe constatar que incluso si aceptamos que el hombre ha ido a la Luna, si en la comida familiar nuestro primo nos dijera que había estado allí, pero que no puede probarlo, no lo entenderíamos, consideraríamos que está loco o que quiere gastarnos una broma. Si por el contrario, lo demostrara con evidencias empíricas contrastables, en apariencia fiables, y explicaciones científicas y contextuales, podríamos empezar a creerlo. Ahora bien, tanto si lo creemos como si no, ya estamos realizando ciertas inferencias y juicios, en cuyo trasfondo se encuentran unas certezas que no cuestionamos y que no necesitamos comprobar, como la existencia de la Tierra, de la Luna y de la fuerza de la gravedad, que puede sustraerse gracias a los avances de la técnica aeroespacial. Todo ello, las preguntas que planteamos a nuestro primo, las inferencias que realizamos, la confianza en sus explicaciones, es posible porque ya compartimos una serie de certezas y formamos parte de una comunidad unida por la ciencia y la educación (SC \$298). Si no compartiésemos esta base común, el debate con nuestro primo sería completamente distinto al escenario que acabo de plantear. Por ejemplo, dar evidencias científicas y pruebas del viaje de nuestro primo a la Luna no serviría de nada para argumentar con un miembro de una comunidad que cree que los hombres van a la Luna cuando sueñan pero no cree que se pueda llegar al satélite con una 
nave espacial ${ }^{21}$ (SC \$106). Es decir, la forma de dar evidencias y creer en pruebas varía respecto de las comunidades, así como a lo largo del tiempo, lo que vuelve a ser una muestra de que la prueba definitiva que proponía Moore tampoco funciona en todos los casos.

En conclusión y como ya se explicó en la primera parte de este artículo, según el segundo Wittgenstein las certezas cambian tanto a lo largo del tiempo como en distintos juegos de lenguaje, y entonces también cambian los conceptos y los significados de las palabras (SC \$62). Para explicar este proceso, Wittgenstein utiliza la metáfora de un río, donde ciertas proposiciones, las certezas, en un momento son sólidas y forman el cauce y posteriormente pueden fluidificarse e integrarse en el cuerpo de las proposiciones empíricas, que fluyen como el agua (SC \$96). En nuestro caso, la frase «ningún ser humano ha ido a la Luna» ha pasado de ser una certeza incuestionada a ser falsa; pero para que se comprenda y se compruebe sigue siendo necesario un cauce, esto es, otras certezas. En respuesta a la pregunta planteada, cabe mantener entonces que la transformación de una certeza en una proposición empírica no elimina las certezas, sino que las desplaza a otras proposiciones y actitudes, esto es, cuando una certeza se transforma en una proposición empírica, van a apareciendo otras proposiciones que creemos, sin necesidad de comprobación, que son las nuevas certezas. Es decir, la relación entre proposiciones empíricas y certezas cambia; sin embargo, siempre distinguimos entre unas proposiciones y otras, "por mucho que no haya una distinción precisa entre una cosa y la otra» (SC $\$ 97$ ).

A continuación y para acabar este análisis, queda contemplar la actitud escéptica de personas que siguen dudando de contenidos que parecen estar comprobados.

\subsubsection{Persistencia de la duda escéptica}

Como ya se ha explicado en los apartados anteriores, la duda escéptica sobre los viajes a la Luna puede persistir. Ya no se trata sólo de la abuela que cuestiona, en el salón familiar, que su nieto haya visitado la Luna; sino del conjunto de personas que aún hoy en día no cree que Armstrong fuera a la Luna, porque considera que todo fue un montaje propagandístico, orquestado por Kubrick en Hollywood, para ensalzar la moral de EE. UU. y adelantar a la URSS en la carrera espacial. Este grupo

${ }^{21}$ No deseo continuar con las diferentes creencias y formas de comprobación de distintas culturas porque me alejaría del contexto cotidiano tratado aquí. Considero, sin embargo, que cuando Wittgenstein se refería a otras formas de vida con otras creencias (por ejemplo, SC \$106 y $\$ 264$, IF \$200-206) no pretendía realizar consideraciones antropológicas sobre las diferencias culturales, como, por ejemplo, mantiene Peter Winch (Comprender una sociedad primitiva, Barcelona, Paidós, 1994), sino que estaba proponiendo ejemplos para pensar qué papel juegan las certezas en los juegos de lenguaje y cómo pueden cambiar. Si se quiere leer más sobre este posible diálogo entre un miembro de la cultura occidental y un miembro de una cultura que no crea que el ser humano ha ido a la Luna, se puede consultar el artículo de Analisa Coliva "Was Wittgenstein an Epistemic Relativist?», Philosophical Investigations 33, enero 2010 (pp. 1-23). 
de escépticos trae a colación sus propias razones y evidencias para justificar su duda o descreimiento; por ejemplo, la bandera de EE. UU. que colocó Armstrong en el satélite ondeaba, pero ¿cómo iba a ser esto posible si en la Luna no hay atmósfera y, por consiguiente, no hay viento?

Por mucho que los científicos den respuesta a esta cuestión (y a otras muchas), quien siga desconfiando de la posibilidad de viajar a Luna, lo seguirá haciendo, apoyado por otros que piensan igual, confían en las mismas fuentes y ofrecen sus propias evidencias. Además, esta posibilidad de corroboración de la duda escéptica resulta más fácil hoy en día por la cantidad de datos y opiniones diversas que se pueden encontrar en Internet. Es decir, aunque la llegada del hombre a la Luna sea (para muchas personas) un hecho empírico mostrado y posibilitado por el avance de la ciencia aeroespacial, sigue siendo un conocimiento que no obtenemos directamente, sino que depende de la confianza que ponemos en ciertos sujetos y en ciertas fuentes (en los libros de texto, en la ciencia aeroespacial, en los medios de comunicación...). Ahora bien, si alguien no confiara en tales fuentes y optara por las suyas propias, o por las de otras comunidades; por ejemplo, si creyera más lo que lee en ciertos foros de escépticos en Internet que en lo que dicen los libros de texto y nos recordara que Wikipedia tiene una entrada dedicada a explicar cómo se hizo el montaje ${ }^{22}$, ¿cómo podríamos convencerle o hacerle cambiar de opinión?, ¿cómo sería posible argumentar con él?, ¿qué evidencias le podríamos ofrecer para refutar su escepticismo? Prácticamente ninguna, ya que como en el caso planteado anteriormente de una comunidad no occidental que no conoce el avance de la técnica aeroespacial, por lo que no cree que el hombre haya ido a la Luna (SC \$106ss.), esta comunidad de escépticos también tiene su propio sistema de creencias, sus propias certezas y actitudes y está unida ya no tanto por la ciencia y la educación, sino por la teoría de la conspiración, el escepticismo y la crítica a EE. UU., que ha logrado que su versión de la historia del siglo xx se entienda como «la verdad». La proposición «ningún ser humano ha ido a la Luna» sería verdadera para ellos y tendrían la certeza de que nadie ha ido a la Luna. Esta comunidad también tendría un modo de actuar común; por ejemplo, se reunirían en fechas acordadas por el calendario habitual, donde seguirían aduciendo motivos de su escepticismo (por ejemplo, las fotos estaban trucadas) y tratarían de convencer a otros con sus argumentos y explicaciones, dando otras fuentes y proponiendo sus propias teorías escépticas.

Cabe entonces concluir, como hace Wittgenstein, que incluso en el caso del escepticismo se han de mantener algunas certezas (SC \$115), si se pretende que sus dudas sean comprensibles, con independencia de que se compartan o no. Esto se debe a que no se puede comprobar todo («quien no está seguro de ningún hecho tampoco puede estarlo del sentido de sus palabras» SC \$114), y a que hay que dar algo por supuesto (qué sujeto cuenta como fiable, qué admitimos como evidencia, qué es una duda válida...) para comenzar a dudar y preguntar; en definitiva, para

${ }^{22} C f$. http://en.wikipedia.org/wiki/Moon_landing_conspiracy_theories [última consulta: 29 de enero de 2018]. 
tratar de entendernos. Pese a su escepticismo, este grupo de personas también tendría que admitir alguna certeza, como su propia existencia y la de los otros seres humanos (si no, ¿cómo iban a entenderse y ponerse de acuerdo o discutir?), o que usan un mismo idioma para comunicarse y que cuando dicen «Luna» se refieren al satélite de la Tierra, no a una plaza de Madrid. ¿Es necesario explicitar todos estos contenidos en forma de proposiciones que tengan la forma "sé que...»? De nuevo, no, porque se trata de las certezas que permiten la articulación de estas dudas y posibilitan el entendimiento entre los miembros de esa comunidad, así como su comunicación con otras personas.

Sin embargo, y aunque sea comprensible y se dé en algunos casos, la duda escéptica sobre los viajes a la Luna tampoco es muy significativa o relevante, ya que si un grupo de personas dudara de que el hombre ha viajado a la Luna y adujera razones y evidencias para mostrar el porqué de esas dudas, ¿cómo afectaría su escepticismo a su actividad cotidiana? Seguramente discutirían y darían sus razones, y en el ejemplo de la comida familiar no creerían a nuestro primo, considerarían por ejemplo que sus fotos estaban trucadas. Exceptuando esta posibilidad ( $\mathrm{y}$ otras similares), ¿qué actitud tendrían estos escépticos cuando van al supermercado o al cine? Probablemente la misma que todos los que formamos parte de esta comunidad. Esto es, a pesar de su duda sobre la llegada del ser humano a la Luna, el escéptico seguiría manteniendo el ciclo de los días y las noches, preparando su comida, trabajando, durmiendo; quizás esté atento a la subida de las mareas si vive cerca del mar y hay aviso de tormenta tropical y puede que, incluso, algún día se maravillara por la contemplación de una Luna perfectamente llena, quizás no; pero, en definitiva, el carácter verdadero o falso de la llegada del hombre a la Luna apenas afectaría a esta persona en su vida cotidiana. Su duda sería lo que Wittgenstein llamó una duda «ociosa» (SC \$117), que se da en el vacío, sin conexión con nada más. En estos casos, se le podría dejar dudar tranquilamente al escéptico, sin que nada pasara, sin que afectara a su quehacer cotidiano (SC $\$ 120$ ).

Ahora bien, ¿y si esta duda escéptica no se refiriera al espacio exterior sino a algo más cercano y cotidiano?, ¿podría seguir dándose? En este caso nos podemos imaginar, como hace Wittgenstein, a alguien que debe recoger a un amigo en la estación de trenes y que, tras consultar el horario, se dice: «No creo que el tren vaya a llegar, pero a pesar de todo, iré a la estación». Es decir, esta persona seguiría haciendo lo mismo que haría si pensara que el tren fuera a llegar (tomar un transporte, ir a la estación con algo de antelación, informarse, esperar en el andén...), pero su duda constante le produce, nos dice Wittgenstein, mal humor (SC \$339). En el caso de que hubiera motivos reales para mantener esta duda (por ejemplo, la poca puntualidad de los trenes, las malas condiciones de las vías, una tormenta eléctrica o una huelga ferroviaria...) seguirían existiendo límites para plantear dudas, no tanto por razones teóricas sino por la práctica cotidiana y compartida de los juegos de lenguaje, de la vida en común. Esto es, si alguien dudara siempre de la fiabilidad del transporte público o de la existencia de las carreteras, apenas podría viajar o moverse más allá de lo que alcanzara a llegar a pie. Su actitud sería poco práctica y difícil de mantener, especialmente en una gran ciudad; además, probablemente también recibiría la incomprensión de sus conciudadanos. Nos encontramos, de nuevo, ante 
dudas «ociosas», poco razonables y poco prácticas, que no se insertan fácilmente en nuestros sistemas de creencias y se distancian de la realidad cotidiana y del modo de hacer común de una comunidad. Incluso si propusiéramos estas dudas u otras similares con una finalidad determinada; por ejemplo, para describir un trastorno psicológico o explicar el escepticismo en una clase de filosofía, estas dudas no serían del todo reales, se encontrarían algo desconectadas de lo que sucede a nuestro alrededor, de las preocupaciones de la gente de a pie. Se trata, en definitiva, de un tipo de dudas que, según Wittgenstein, no son necesarias, ni siquiera cuando son posibles (SC \$392).

Aparece así un límite a la duda escéptica que (a grandes rasgos y con excepciones) se puede dar si no perturba la práctica cotidiana y el modo de hacer común de un grupo humano. Ahora bien, incluso cuando se da, ya supone la confirmación de una serie de certezas. Es decir, incluso si alguien dudara de la posible llegada de los trenes a la estación, seguiría creyendo estar en un huso horario y no en otro (por ejemplo, en la hora del Pacífico), en una cierta ciudad, que existen los demás seres humanos que esperan en la estación y que algunas proposiciones empíricas se pueden corroborar. Dudar de estas certezas supondría muchas dificultades e incluso acercaría a este sujeto a la locura (SC \$421). Ahora bien, jesto quiere decir que estas certezas se conocen del mismo modo como sabemos que Lima es la capital de Perú y que si tocamos el fuego nos quemamos? No, y regresando al ejemplo del viaje a la Luna, aunque haya personas que puedan seguir dudando de ello, su duda escéptica tampoco elimina el papel que cumplen las certezas en los juegos de lenguaje, que pueden cambiar de contenido, pero resultan imprescindibles, como marco, base o eje $^{23}$, para que se dé conocimiento empírico y nos entendamos al hablar con otras personas. Como mantuvo Wittgenstein: «Quien quisiera dudar de todo, ni siquiera llegaría a dudar. El mismo juego de la duda presupone ya la certeza» (SC \$115).

\section{CONCLUSIONES}

Como se ha explicado en este artículo, según el segundo Wittgenstein y a diferencia de la comprensión absoluta de las certezas que daba Moore, el hecho de que una proposición sea certeza o conocimiento empírico depende del contexto, de la finalidad o del uso que se le dé; además, el contenido de las certezas puede cambiar a lo largo del tiempo y las evidencias que se puedan dar para probar el conocimiento empírico también pueden variar según el juego de lenguaje en el que nos encontremos. A pesar de estos matices, consideraciones y posibles cambios, siempre van a seguir dándose algunas certezas, de las que no poseemos evidencias

${ }^{23}$ Estas son algunas de las metáforas que Wittgenstein usa para describir a las certezas (la del marco aparece, por ejemplo, en SC $\$ 86$, la del suelo en SC $\$ 248$, entre otras, y la de los ejes o goznes en SC \$341-343). Como se verá en la conclusión y siguiendo a Moyal-Sharrock (op. cit.), considero que la metáfora del eje es más aclaratoria y adecuada, porque evita una posible comprensión estable y fundacionalista del conocimiento. 
fehacientes porque se basan, más bien, en confiar en ciertas fuentes, en no formular ciertas dudas y en mantener un modo de actuar común con otros seres humanos. De este modo, si alguien dijera que no está seguro de algo que es una certeza en nuestro juego de lenguaje; por ejemplo, que no sabe si tiene cerebro dentro de su cabeza (SC $\$ 4, \$ 118$ ), nos costaría mucho comprender lo que esta persona pretende decir. Esta dificultad de comprensión no se debe a que tengamos un conocimiento seguro que podamos probar y demostrar con evidencias, como Moore proponía, sino a que tenemos un modo de actuar común y a que ciertas dudas (como la de si estamos sobre la Tierra o tenemos cerebro dentro de nuestras cabezas) bloquean la capacidad de comprensión mutua e invalidan el modo en que planteamos preguntas y damos respuestas en los juegos de lenguaje.

Ante la persona que duda de tener cerebro, podríamos proponerle que fuera a un hospital para hacerse un tac craneal o que se abriera una brecha en la cabeza y mirara, pero tanto el planteamiento de esta duda como las soluciones propuestas resultan extrańas, poco necesarias y ligeramente grotescas, nos faltarían razones para plantearlas y se distanciarían mucho de lo que estamos acostumbrados a dudar, a preguntar y responder. Esto no quiere decir que sepamos que tenemos cerebro dentro de nuestras cabezas del mismo modo que sabemos que el símbolo químico del cloro es $\mathrm{Cl}$, sino que no entenderíamos a quien manifestara una duda así.

A partir de consideraciones como estas y de la constatación de que hay preguntas y dudas que alteran el funcionamiento habitual de los juegos de lenguaje (¿cómo sabes si tienes diez dedos de los pies cuando no los miras?, ¿cómo sabes si existe Estambul, si nunca fuiste?, ¿cómo sabes si los muebles desaparecen cuando no estamos en una habitación?), Wittgenstein mantiene que las preguntas y dudas que hacemos descansan sobre otras proposiciones que, aunque no podamos corroborar, están fuera de duda, ya que constituyen los ejes sobre los que gira nuestra comprensión de la realidad (SC \$341). Estos ejes, las certezas, pueden cambiar, de tal modo que aunque para Wittgenstein no era concebible que el ser humano pudiera ir a la Luna, con el avance de la técnica aeroespacial esto se ha logrado y el estatus de esta familia de certezas ha cambiado. Sin embargo, esto no significa la desaparición de estas certezas, sino su modificación. Actualmente, ciertas personas (como Neil Armstrong, Buzz Aldrin y el primo viajero, en nuestro ejemplo) pueden asegurar que han estado en la Luna y su expresión será verdadera, aunque algo extraña. Al mismo tiempo, la expresión «sé que he estado en la Luna» es posible, comprensible y comprobable en la actualidad porque hay otras proposiciones que no se cuestionan, que funcionan como certezas; por ejemplo, la existencia de la Luna y de la Tierra antes de 1969.

Aun así, ciertas personas van a seguir dudando de la posibilidad de los viajes espaciales y darán sus propias razones, argumentos y evidencias para mostrar la imposibilidad de tal viaje. Y probablemente los escépticos nunca lleguen a ponerse de acuerdo con los que sí creen que el hombre llegó a la Luna. Sin embargo, al tratarse de un tema que apenas afecta a nuestra vida cotidiana, esta duda escéptica no sería demasiado problemática, podríamos dejarla estar, quizás como un recordatorio de que gran parte del conocimiento que tenemos y que ponemos en práctica cotidianamente no depende de evidencias que alguien pueda contrastar en su día a día, sino que lo 
hemos adquirido a partir de fuentes en las que creemos (documentales, libros de texto, enciclopedias...) y lo mantenemos porque no contradice nuestra experiencia cercana y porque lo compartimos con otros miembros de nuestra comunidad.

En resumen, para que haya conocimiento, duda y nos entendamos cuando hablamos, siempre va a haber contenidos o ejes que permanecen firmes e incuestionados, aunque carezcamos de evidencias para su demostración, y esto es lo que Wittgenstein entendía como certezas (SC \$342-343). Estas certezas no son absolutas ni siempre iguales, como aducía Moore, sino que cambian dependiendo de los distintos usos que se den del lenguaje, del contexto, del momento o de la finalidad o intención de los hablantes; pero en cualquier caso, la diferencia entre conocimiento y certeza se va a mantener. Volviendo al ejemplo analizado en este artículo, el alunizaje de Armstrong en 1969 cambió el estatus de algunas certezas relacionadas con la presencia del ser humano en el espacio exterior, de tal modo que proposiciones como "el hombre ha ido a la Luna» se han integrado en el conjunto de conocimientos que aparecen en los libros de texto y se aprenden en la escuela (como que el Everest es el pico más alto del mundo o que Napoleón fue vencido en la Batalla de Waterloo), que conocemos porque formamos parte de una misma comunidad, unida por la ciencia y la educación (SC \$298). Ahora bien, estos conocimientos siguen presuponiendo certezas, que no comprobamos (o no podemos comprobar) pero que si cuestionamos causan dificultades en nuestra comprensión. Por ejemplo, podemos consultar en una enciclopedia la biografía de Napoleón, pero eso ya se hace presuponiendo algo que no se comprueba (SC \$163), como que Francia existía en aquella época o que la Tierra tiene, al menos, tantos años de antigüedad como el general francés. De modo similar, también nos resultaría muy extraño que alguien dijera: «Sé que Armstrong alunizó en 1969, pero no sé si la Luna existe realmente o si es sólo una mancha en el cielo». Si alguien expresara dudas como esta, no sabríamos muy bien qué quiere decir, apenas podríamos argumentar con esta persona, ni darle explicaciones o evidencias; quizás podríamos pensar que no sabe utilizar correctamente los verbos «dudar» y «saber», que está aprendiendo a hablar castellano, que nos quiere engañar de alguna manera o que se ha confundido de alguna manera que no llegamos a entender del todo... Estas dificultades de comprensión son una muestra de que esta persona está intentando dudar o cuestionar una certeza de nuestro juego de lenguaje, que aun sin evidencias, no podemos evitar mantener, si es que pretendemos interactuar y entendernos con los demás seres humanos que nos rodean ${ }^{24}$.

Recibido: febrero de 2018, aCEPTAdo: junio de 2018

${ }^{24}$ Siguiendo la crítica del segundo Wittgenstein a la posibilidad de encontrar y fijar un único uso del lenguaje, no desearía que este artículo se entendiera como una explicación general y abstracta sobre el funcionamiento del lenguaje, sino como una descripción del papel que juegan algunas certezas en el conocimiento cotidiano. 
